\title{
EDITORIAL
}

\section{Freedom from chronic lung allograft dysfunction (CLAD) or CLAD-free survival: What's in a name?}

\author{
Geert M. Verleden, MD, PhD, ${ }^{a}$ Stijn E. Verleden, $\mathrm{PhD},{ }^{\mathrm{b}}$ and \\ Robin Vos, MD, PhD
}

From the ${ }^{a}$ Lung Transplantation Unit, University Hospital Gasthuisberg, Leuven, Belgium; and the ${ }^{b}$ Dept Chrometa, Lung Transplantation Unit, Katholieke Universiteit Leuven, Leuven, Belgium.

Long-term outcomes after lung transplantation (LTx) remain inferior to those of other solid organs, such as heart transplantation. This is largely the result of a high prevalence of chronic lung allograft dysfunction (CLAD), which affects approximately $50 \%$ of transplanted patients at 5 years post-transplant. ${ }^{1}$ Although bronchiolitis obliterans syndrome (BOS) is the major clinical phenotype of CLAD (occurring in $70 \%$ of CLAD patients), restrictive allograft syndrome (RAS), the other CLAD phenotype (30\%), has shown worse survival after diagnosis. ${ }^{2}$ Whereas the median post-BOS survival is between 3 and 5 years, ${ }^{3}$ the outcome after RAS is limited to 1 to 2 years. ${ }^{4}$

Actuarial survival and freedom from BOS have so far been considered as the discrete outcomes of greatest interest after LTx. However, we believe that discretely separating these entities may underestimate the real contributions of BOS. As an example, a patient may develop BOS before death, or may die before a diagnosis of BOS. To avoid this pitfall, in this issue of the Journal, Kulkarni et $\mathrm{al}^{5}$ describe BOS-free survival as a composite end-point taking into account BOS development and death, thus assessing the implied contribution of both of these facets rather than discretely reporting outcomes. The authors used the Registry database of the International Society for Heart and Lung Transplantation (ISHLT) to produce these data in a cohort of $>15,000$ patients transplanted between 1994 and 2011 (with follow-up until 2012). Whereas the Registry report suggests a 5-year freedom from BOS of 50\%, ${ }^{1}$ Kulkarni et al found that 5-year BOS-free survival was only around $36 \%$ and median BOS-free survival 3.5 years, varying between 3.58 and 4.00 years for bilateral lung transplants and 3.16 to 3.19 years after single-lung transplantation. The

Reprint requests: Geert M. Verleden, MD, PhD, Lung Transplantation Unit, University Hospital Gasthuisberg, 49, Herestraat, B-3000 Leuven, Belgium. Telephone: 32-16-346805. Fax: 32-16-346803.

E-mail address: geert.verleden@uzleuven.be
post-BOS diagnosis survival was found to be 3.8 to 4.3 years after bilateral and 2.6 to 3.1 years after singlelung transplantation. ${ }^{5}$ This is a relatively sobering new presentation for lung transplant outcome that deserves reflection and critical analysis.

There are drawbacks to the analysis that make it difficult to clearly interpret the results. The authors included $<50 \%$ of all transplanted patients from whom BOS data were available, and $>99 \%$ of them were from North America, indicating a geographic bias in reporting this outcome (as the diagnosis may be made variably in other regions or patient cohorts may have variable incidences). Indeed, there are no multicenter BOS data available from Europe and Australia. Importantly, a registry includes data from multiple centers, representing those with a high volume and more experience as well as others with low volume and less experience. Therefore, such multicenter registries may tilt results toward very experienced centers due to a reporting bias based on volume. Moreover, the data on BOS accumulated in the registry imply presence or absence of a diagnosis of BOS, such that the exact date of onset is somewhat uncertain and no quantitative phenotype expression of the presentation is identified (BOS, RAS, or mixed). Diagnosing CLAD is not always simple because of other disease conditions that may concur with its development, such as bronchial stenosis, myopathy, acute rejection, and acute infection. This may not only impact the exact timing of CLAD onset, but may also challenge the diagnosis. This was clearly demonstrated in a study by Kapila et al, where only $70 \%$ agreement on the diagnosis of BOS was noted. ${ }^{6}$ Last, BOS constitutes only $70 \%$ of all CLAD cases, meaning that CLAD-free survival would be even worse if RAS was also included.

Although the Kulkarni et al findings are sobering, we should contrast their results with data emerging from single, high-volume, experienced centers to gain an understanding of the potential of better outcomes after LTx. At our own 
center (one of the largest and experienced centers worldwide) the median survival after LTx is 11.1 years, compared with 7.4 and 4.6 years noted in the ISHLT Registry, respectively, for double- and single-lung transplantation. More recently, our 5-year survival has increased to $>75 \%$, compared with $55 \%$ in the Registry analysis. ${ }^{7}$ Our 5 -year freedom from CLAD is around 70\%, compared with 50\% in the Registry. ${ }^{8}$ Is not fully understood why these results are different; however, we use a longitudinal electronic database (which allows little information loss), close follow-up, so no patients are lost to follow-up, and we expose patients to a standardized diagnosis and medical treatment of CLAD.

The limitations of registry-based data analyses are not unique. Such limitations were recently also encountered in the ISHLT DCD LTx Registry. ${ }^{9}$ The current BOS data within the Registry need further refinement with better phenotyping of CLAD and a well-defined time of onset of CLAD. Information on treatment options would also be very helpful, especially for guiding future therapy trials. Before this can be done, we need consensus on the definitions of CLAD and RAS, and this is best accomplished as an international project under the aegis of the ISHLT, by harnessing the combined talent within the pulmonary community. Only by optimizing data reporting and working together through consensus will we be able to continually enhance the LTx outcome, for which CLAD-free survival may become a standard parameter for determination of therapeutic success.

\section{Disclosure statement}

The authors have no conflicts of interest to disclose.

\section{References}

1. Chambers DC, Yusen RD, Cherikh WS, et al. The Registry of the International Society for Heart and Lung Transplantation: thirty-fourth adult lung and heart-lung transplantation report-2017. Focus theme: Allograft ischemic time. J Heart Lung Transplant 2017;36:1047-59.

2. Verleden GM, Raghu G, Meyer KC, et al. A new classification system for chronic lung allograft dysfunction. J Heart Lung Transplant 2014;33:127-33.

3. Verleden SE, Sacreas A, Vos R, et al. Advances in understanding bronchiolitis obliterans after lung transplantation. Chest 2016;150:219-25.

4. Sato M, Waddell TK, Wagnetz U, et al. Restrictive allograft syndrome (RAS): a novel form of chronic lung allograft dysfunction. J Heart Lung Transplant 2011;30:735-42.

5. Kulkarni HS, Cherikh WS, Chambers DC, et al. Bronchiolitis obliterans syndrome-free survival following lung transplantation-an International Society for Heart and Lung Transplantation (ISHLT) Thoracic Transplant Registry analysis. J Heart Lung Transplant 201X;XX:000-00.

6. Kapila A, Baz MA, Valentine VG, et al. Reliability of diagnosic criteria for bronchiolitis obliterans syndrome after lung transplantation: a survey. J Heart Lung Transplant 2015;34:65-74.

7. Van Raemdonck D, Neyrinck A, Vos R, et al. Living by numbers. J Thorac Cardiovasc Surg 2018;156:906-7.

8. Ruttens D, Verleden SE, Vandermeulen E, et al. Prophylactic azithromycin therapy after lung transplantation: post hoc analysis of a randomized controlled trial. Am J Transplant 2016;16:254-61.

9. Levvey B, Keshavjee S, Cypel M, et al. Influence of lung donor agonal and warm ischemic times on early mortality: analyses from the ISHLT DCD lung transplant registry. J Heart Lung Transplant, in press. 